\title{
MEGGER AND OTHER TESTS ON SUSPENSION INSULATORS
}

\author{
BY F. L. HUNT
}

\section{Abstract of Paper}

This paper gives the results of megger tests made on disk insulators on a 66,000-volt transmission line in central Massachusetts after 2.5 years' operation. In one lot of 4410 insulators tested 9.77 per cent tested less than 2000 megohms. The percentage of failures in different positions in the string is given on both strain and suspension towers. The actual cost of making these tests under different conditions of weather and of service requirements is given per insulator on the line, per bad insulator and per tower. The cost per insulator on the line, of testing only, varied from 7.3 cents to 11 cents. The cost of replacing bad insulators was 74.5 cents per bad insulator, not including the cost of the replacing insulator.

Laboratory tests made on 30 of the bad insulators taken from the line showed that those which measured very low by the megger fail on 60-cycle tests much below spark-over value, while those with medium high resistance may reach flash-over value and then puncture within one minute. Some insulators which show infinite resistance by the megger, and which withstand arc-over potential at 60 cycles, will fail under high-frequency test immediately.

URING the latter part of 1915 , megger tests were made on all the insulators on one circuit, and part of the insulators on the other circuit, on nineteen miles of 66,000 -volt, double circuit transmission line in central Massachusetts. These circuits are supported on galvanized steel towers, three wires to each circuit spaced $10 \mathrm{ft}$. (3m.) apart vertically, the two circuits spaced $18 \mathrm{ft} .(5.4 \mathrm{~m}$.$) apart horizontally, the bottom wire 45 \mathrm{ft} .(13.7 \mathrm{~m}$. from the ground, average span, $550 \mathrm{ft}$. $(167.6 \mathrm{~m}$.)

The total number of insulators tested on this line was 4410 . These were suspension type insulators hung with four per wire on suspension towers and five per wire on dead end towers. Numbering the insulators from 1 to 5 , beginning at the cross arm, the number of failures in each position is shown in the following tabulation. 


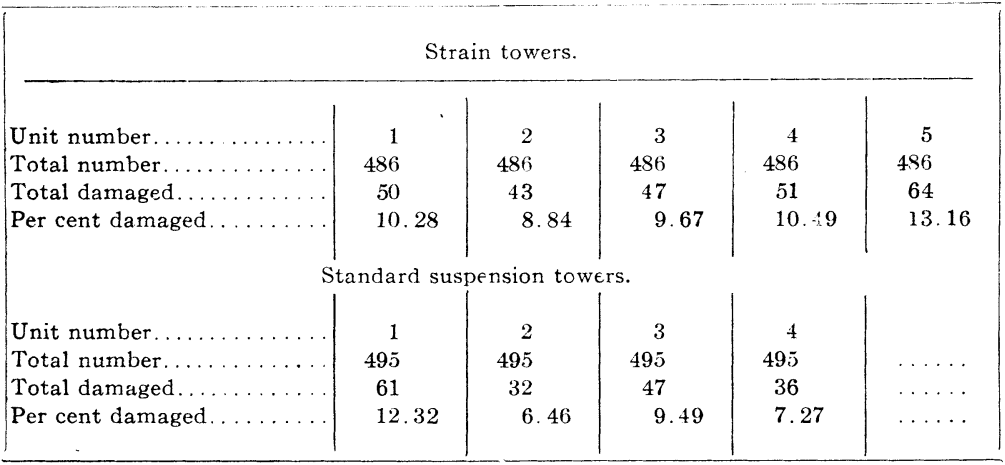

The above results seem to indicate that there was no logical relation between the number of failures of the insulators in any given position and their distance from the conductors.

\section{Cost of Tests}

It was necessary in testing these insulators to take one circuit at a time, so that all the towers had to be gone over twice. Of the total number of 4410 insulators tested, there were 2430 dead end insulators, of which 255 , or 10.5 per cent, were bad; and 1980 suspension insulators, of which 176 , or 8.9 per cent were bad, as indicated by megger tests above.

A crew of three men was used in making the tests and twenty week days and twelve Sundays were consumed.

The total cost of the tests, including cost of transportation, was $\$ 424.69$.

The insulators on one circuit were tested on 180 towers and on the other circuit on 69 towers. The cost per tower per circuit on the first 180 towers was $\$ 1.97$. The cost per tower per circuit on the 69 towers was $\$ 1.02$ each. The average cost of the whole job was therefore $\$ 1.70$ per tower per circuit, and 9.6 cents per insulator tested, where only one circuit could be tested at a time with the other circuit alive during the testing.

The variation in cost per tower in the two parts of the job was due almost entirely to the difference in weather conditions. In the first case the weather was cold and the insulators were covered with frost, so that the work could not be started until about ten o'clock in the morning. The men were also stopped several times by rain, so that considerable time was lost, and this section of the testing included most of the Sunday work.

The total cost of replacing the 431 bad insulators was $\$ 322.27$ or 7.3 cents per insulator on the line and 74.5 cents per bad in- 
sulator. The total labor cost of testing and replacing bad insulators on the line was therefore 16.9 cents per insulator of the total number or $\$ 1.73$ per bad insulator. This does not cover the cost of new insulators. If it had been necessary to pay for new insulators, the total cost per bad insulator would have been three times the original cost.

These insulators were in use less than three years, having been installed in the early part of 1913, put into operation in May, 1913, and tested in the latter part of 1915.

A recent inquiry made from an insulator manufacturing company brought out the fact that they would add 4 cents per disk to the price of the insulators in order to cover the cost to them of putting their insulators through a high-frequency test before shipment. If such a test would have taken out the insulators that have failed during the first 2.5 years of operation, it would appear that 4 cents additional in the initial price would have been a good investment, since the cost of testing and replacing bad insulators has amounted to 16.9 cents per disk on the line.

Another section of line of about the same length upon which insulators of a different design had been installed, was tested at a cost of 7.3 cents per insulator, which is slightly less than the cost per insulator in the first case. Due to the fact that both circuits were tested at the same time, each tower had to be climbed but once. The insulators in this second case showed less than one per cent of failures and, on this account, it was not considered necessary to replace the bad insulators, since there was no case in which more than one bad insulator occurred in one string.

\section{LABORATORY TESTS}

To get definite information regarding the nature of the faults in the insulators they were shipped to a laboratory where the following tests were made with the co-operation of Mr. E. E. F. Creighton and Mr. P. E. Hosegood.

The total number of insulators selected was 40 . Thirty-five of these were taken from the line and five were new insulators. Twenty-one measured below 10 megohms, eight measured 22 to 52 megohms; one measured 220 megohms, five from the line measured infinity, and the five new insulators measured infinity, making up the total of 40 .

Test 1. Eleven of the insulators which meggered below 10 megohms had 60 -cycle voltage gradually applied to them until they punctured. Not one of them reached flash-over value. 
The voltage was very gradually raised and the lowest puncture voltage was $22 \mathrm{kv}$., the highest was $52 \mathrm{kv}$., and the average of the 11 was $39 \mathrm{kv}$.

Test 2. Seven of the insulators which measured between 22 and 52 megohms and the one measuring 220 megohms had arcover voltage applied to them until they punctured. Four of them punctured immediately, 2 of them punctured in one second, one in four seconds, and one in thirty seconds. There is no relation between the megger readings and the time of puncture.

Test 3. In this test a constant volta ge far below the arc-over value was applied until puncture occurred. Seven insulators measuring below 10 megohms were tested. The first five had applied to them $20 \mathrm{kv}$. They punctured in from one to thirty seconds. Two more were tested at $15 \mathrm{kv}$., one puncturing in one second, the other in six seconds. There is no consistency between the puncture voltage and the megger readings as sufficiently illustrated by the last test. In this test one of the insulators measured three megohms and the other below one megohm, but the higher resistance insulator took one second to puncture whereas the other insulator with lower resistance took six seconds to puncture.

Test 4. Spark-over voltage of 60 cycles was applied to the 10 insulators which measured infinity. Three of these punctured in less than 45 seconds. This is 30 per cent loss on 60 cycles.

Test 5. The remaining seven insulators which had shown O.K. on a minute's test on 60 cycles at flash-over voltage, were next tested on the oscillator using a frequency of approximately 200,000 cycles per second. Flash-over voltage only was applied. Two of the seven punctured in less than one minute. This is again about 30 per cent of the insulators that were left. The remaining five withstood super-spark potential of $120 \mathrm{kv}$. for 10 seconds each.

Conclusions. The results of these tests are similar to others that have been made and show, first, that insulators which measure very low will fail almost immediately on a 60 -cycle test voltage, much less than spark-over value. Insulators with medium high resistance may reach flash-over value of potential and will puncture within a minute. Insulators of infinite megger reading may have faults developed by the arc-over potential within one minute's application. After the faulty insulators have been eliminated by the 60 -cycle test, still further faults are found by the oscillator. 